tumours: a retrospective cohort study. Lancet. 2012;380(9840):499505

8. Fernandez-Antoran D, Piedrafita G, Murai K, et al. Outcompeting p53-Mutant Cells in the Normal Esophagus by Redox Manipulation. Cell Stem Cell. 2019;25(3):329-341.e6.

9. Rodrigues-Moreira S, Moreno SG, Ghinatti G, et al. Low-Dose Irradiation Promotes Persistent Oxidative Stress and Decreases SelfRenewal in Hematopoietic Stem Cells. Cell Rep. 2017;20(13):31993211.

10. Henry E, Souissi-Sahraoui I, Deynoux M, et al. Human hematopoietic stem/progenitor cells display ROS-dependent long-term hematopoietic defects after exposure to low dose of ionizing radiations. Haematologica. 2020;105(8):2044-2055.

11. Ito K, Hirao A, Arai F, et al. Reactive oxygen species act through p38 MAPK to limit the lifespan of hematopoietic stem cells. Nat Med. 2006;12(4):446-451.

12. Miyamoto K, Araki KY, Naka K, et al. Foxo3a is essential for maintenance of the hematopoietic stem cell pool. Cell Stem Cell. 2007;1(1):101-112.

13. Tothova Z, Kollipara R, Huntly BJ, et al. FoxOs are critical mediators of hematopoietic stem cell resistance to physiologic oxidative stress.
Cell. 2007;128(2):325-339.

14. Balaban RS, Nemoto S, Finkel T. Mitochondria, oxidants, and aging Cell. 2005;120(4):483-495.

15. Nissanka N, Moraes CT. Mitochondrial DNA damage and reactive oxygen species in neurodegenerative disease. FEBS Lett. 2018;592(5):728-742.

16. Kawamura K, Oi F, Kobayashi J. Potential relationship between the biological effects of low-dose irradiation and mitochondrial ROS production. J Radiat Res. 2018;59(suppl_2):ii91-ii97.

17. Youle RJ, van der Bliek AM. Mitochondrial fission, fusion, and stress. Science. 2012;337(6098):1062-1065.

18. Ho TT, Warr MR, Adelman ER, et al. Autophagy maintains the metabolism and function of young and old stem cells. Nature. 2017;543(7644):205-210

19. Ito K, Turcotte $R$, Cui J, et al. Self-renewal of a purified Tie2+ hematopoietic stem cell population relies on mitochondrial clearance. Science. 2016;354(6316):1156-1160.

20. de Almeida MJ, Luchsinger LL, Corrigan DJ, Williams LJ, Snoeck HW. Dye-Independent Methods Reveal Elevated Mitochondrial Mass in Hematopoietic Stem Cells. Cell Stem Cell. 2017;21(6):725729.e4

\title{
Busy signal: platelet-derived growth factor activation in myelofibrosis
}

\section{Anna E. Marneth ${ }^{1}$ and Ann Mullally ${ }^{1,2,3}$}

${ }^{1}$ Division of Hematology, Department of Medicine, Brigham and Women's Hospital, Harvard Medical School, Boston; ${ }^{2}$ Dana-Farber Cancer Institute, Harvard Medical School, Boston and ${ }^{3}$ Broad Institute, Cambridge, MA, USA.

E-mail: ANN MULLALLY - amullally@partners.org

doi:10.3324/haematol.2020.253708

T The pathogenesis of myelofibrosis, a bone marrow (BM) disorder characterized by megakaryocytic hyperplasia and the deposition of extracellular matrix components such as reticulin, remains incompletely understood.

Using a mouse model of myelofibrosis (i.e. Gata-1 $1^{\text {low }}$ mice), Kramer et al. ${ }^{1}$ sought to identify key signaling molecules that play a role in early myelofibrosis development. GATA-1 is a transcription factor that is key to megakaryocyte development, and its downregulation results in expansion and abnormal maturation of megakaryocytes. ${ }^{2}$ Importantly, low GATA-1 expression has been demonstrated in patients with myelofibrosis, ${ }^{3}$ and GATA-1 mutations are found in megakaryocytic leukemias. ${ }^{2}$

\section{New key findings}

Unlike several widely used myelofibrosis mouse models that rely on BM transplantation to engender fibrosis, primary Gata- $1^{\text {low }}$ mice gradually develop myelofibrosis spontaneously. ${ }^{4}$ Due to its slow progression, this model allows for analysis at prefibrotic (5 months), early fibrotic (10 months), and overtly fibrotic (15 months) stages. A strength of the study by Kramer et al. is the application of an unbiased approach (i.e. RNA sequencing) to interrogate the changes that occur in receptor tyrosine kinase pathways during the development of myelofibrosis. Using bulk RNA sequencing on unfractionated BM (including stromal cells), the authors identified the platelet-derived growth factor (PDGF) pathway as significantly up-regulated in early fibrotic Gata- $1^{\text {low }}$ mice compared to wild-type mice. Additionally, the authors analyzed protein expression of PDGF receptors and ligands on $\mathrm{BM}$ sections at the three aforementioned time points; this allowed them to study the PDGF pathway in a spatio-temporal manner.

In addition to demonstrating increased transcript expression of PDGF receptor a (Pdgfra) and Pdgfrb, as well as the ligand Pdgfb, in fibrotic Gata-1 $1^{\text {low }}$ mice, the authors employed a novel technique called in situ proximity ligation assay to determine protein localization. They found that the receptor PDGFR $\beta$ and ligand PDGF-B are in close proximity in the setting of overtly fibrotic $\mathrm{BM}$, suggesting binding of the ligand to the receptor and increased PDGF$\mathrm{B}$ signaling. Furthermore, their data suggest that the most important cell types involved in PDGF signaling are megakaryocytes, which express PDGFR $\alpha$ and secrete the ligand PDGF-B, and spindle-shaped stromal cells which express PDGFR $\beta$ (Figure 1).

Despite these findings, Kramer et al. did not detect increased PDGFR $\beta$ tyrosine phosphorylation, a marker of receptor activation. They suggest that the phosphatase TC-PTP (PTPN2) may play a role in dephosphorylation of PDGFR $\beta$ and show that TC-PTP is in close proximity to PDGFR $\beta$ in fibrotic Gata-1 $1^{\text {low }}$ mice. There are two main potential explanations for these findings. Either: (i) PDGF receptor activation is transient and rapidly down-regulated; or (ii) PDGF receptor activation is rapidly reset by phosphatases such as TC-PTP after ligand binding. Rapid downregulation would call into question the importance of the PDGF pathway in myelofibrosis, while a rapid reset may increase signaling in the presence of ligand and potentially contribute to the development of myelofibrosis. Further investigation of PDGF signaling in human myelofibrosis will be required to fully resolve this question. 


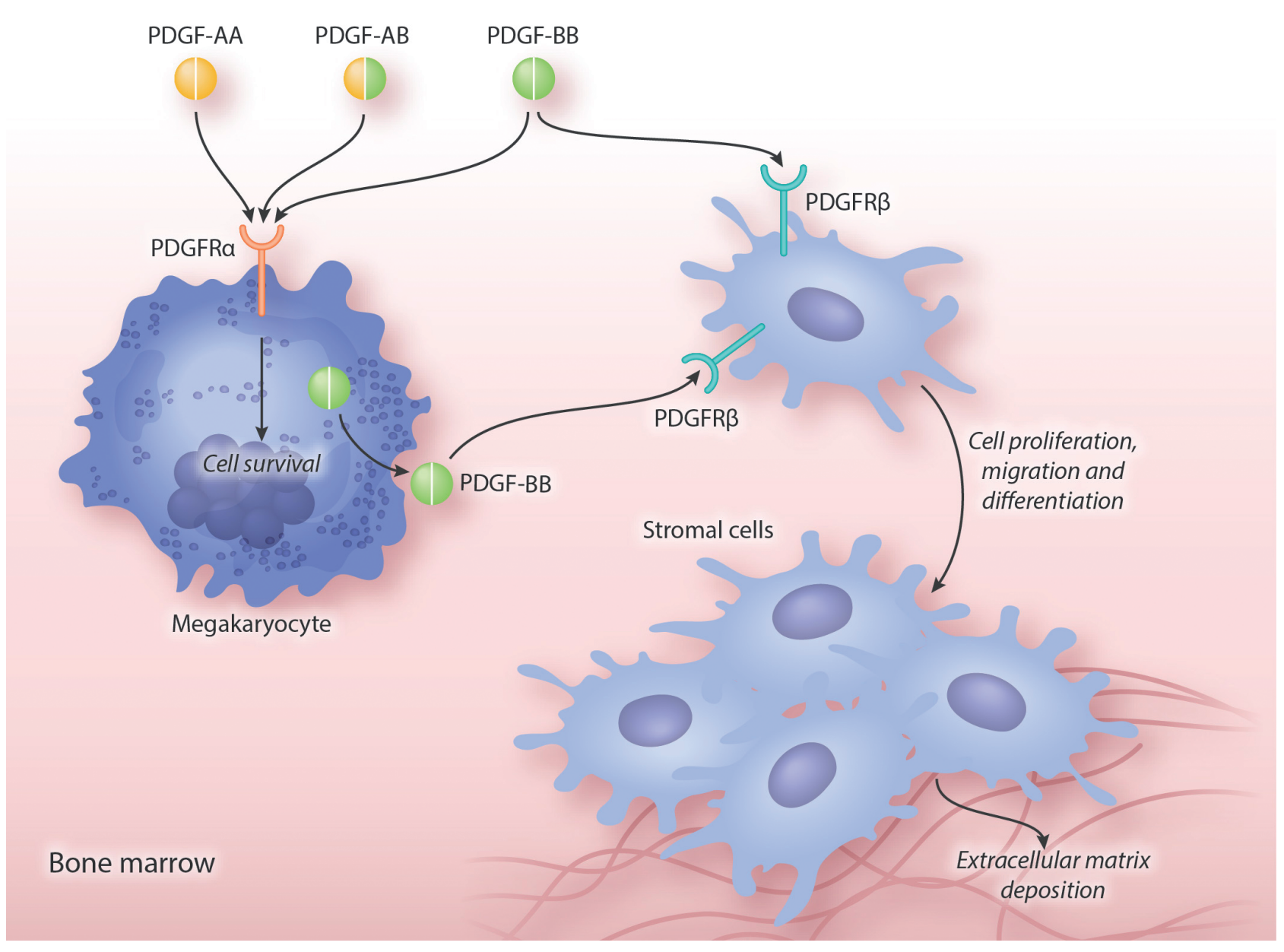

Figure 1. Platelet-derived growth factor (PDGF) signaling in myelofibrosis. Previous (human) data and the study by Kramer et al. ${ }^{1}$ indicate that the ligands PDGF-A and PDGF-B and their receptors PDGFR $\alpha$ and PDGFR $\beta$ play important roles in myelofibrosis development. PDGFR is mainly expressed on megakaryocytes and can be activated by the dimeric ligands PDGF-AA, PDGF-AB, and PDGF-BB. PDGFR $\beta$ is expressed on stromal cells and can be activated through the ligand PDGF-BB. PDGFBB stimulates stromal cell proliferation, migration and differentiation, which in turn causes extracellular matrix deposition and myelofibrosis development.

\section{Platelet-derived growth factor pathway as a potential biomarker for myelofibrosis development}

Platelet-derived growth factors are growth factors for fibroblasts and stromal cells. Importantly, upregulation of the receptors PDGFR $\alpha$ in megakaryocytes and PDGFR $\beta$ in stromal cells, as well as upregulation of their ligands PDGF-A and PDGF-B, has been shown in established human myelofibrosis. ${ }^{5.7}$ Moreover, the grade of myelofibrosis in myeloid malignancies correlates with the level of PDGFR $\beta$ expression in activated fibroblasts. ${ }^{6}$ These data suggest that PDGF signaling contributes to myelofibrosis development.

Myelofibrosis occurs in the context of megakaryocyte disorders, encompassing both inherited bleeding and platelet disorders ${ }^{8,9}$ and myeloid malignancies, most commonly, myeloproliferative neoplasms (MPN). Although patients with MPN can present with de novo myelofibrosis (i.e. primary myelofibrosis, PMF), it can also occur as a complication of antecedent MPN (i.e. post-polycythemia myelofibrosis, PPV-MF or post-essential thrombocythemia myelofibrosis, PET-MF). In patients with PV and $\mathrm{ET}$, it is currently difficult to predict who will progress to myelofibrosis. The study by Kramer et al. raises the ques- tion of whether increased PDGF signaling could serve as an early biomarker for myelofibrosis development.

Bedekocivs et al. ${ }^{6}$ previously assessed PDGFR $\beta$ expression in fibrotic and non-fibrotic BM from several myeloid malignancies and proposed that elevated PDGFR $\beta$ expression could indicate a prefibrotic state. Kramer et al. found increased PDGFR $\alpha$ expression in the prefibrotic stage, but no increase in PDGFR $\beta$ or the ligands PDGF-A and -B. In future studies, it would be informative to measure the dynamics of PDGF components in human myelofibrosis development, using a longitudinal study to determine their predictive and prognostic value.

\section{Targeting the platelet-derived growth factor pathway}

In conjunction with prior (human) studies, this study by Kramer et al. suggests that the PDGF pathway is a potential therapeutic target in myelofibrosis. PDGFR are one of the main targets of the tyrosine kinase inhibitor, imatinib. ${ }^{10}$ Treatment with imatinib has demonstrated clinical benefit in patients with hypereosinophilic syndromes and chronic myeloproliferative disorders who have chromosomal translocations involving PDGFR $\alpha$ and PDGFR $\beta$, respectively. ${ }^{11,12}$ Thrombopoietin (Thpo) is the major stimulant for 
megakaryopoiesis and its overexpression engenders myelofibrosis in mouse models. ${ }^{13}$ Using a Thpo overexpression model, Decker et al. ${ }^{14}$ showed that stromal deletion of PDGFR $\alpha$ or treatment with imatinib suppressed stromal cell expansion and ameliorated myelofibrosis. In addition to imatinib, other methods of targeting the PDGF pathway are currently under investigation in different fibrosis models, such as PDGF/PDGFR-blocking antibodies and aptamers. ${ }^{15}$

\section{Dual targeting of JAK and JAK/platelet-derived growth factor downstream pathways}

Primary myelofibrosis is caused by MPN phenotypic driver mutations (i.e. in JAK2, CALR or $M P L$ ) that result in constitutive activation of JAK-STAT signaling. ${ }^{16}$ Although JAK2 inhibitors such as ruxolitinib reduce constitutional symptoms and splenomegaly, and may stabilize myelofibrosis, they do not have substantial disease-modifying activity in MPN. Inhibiting other tyrosine kinases including PDGF receptors is not sufficient either, since imatinib treatment in PMF was disappointing. ${ }^{17}$ A combinatorial approach involving JAK2 and PDGF inhibition in MPN could be considered, although hematologic toxicity is a real concern. ${ }^{18}$

An alternative treatment strategy involves simultaneously inhibiting JAK-STAT and MEK-ERK signaling. A recent MPN preclinical study showed that JAK2 inhibitors induce a strong reduction in STAT signaling but only marginally reduce MEK/ERK signaling. ${ }^{19}$ Multiplexed analyses of 34 secreted factors in Jak2 V617F-mutant mice showed that transcript levels of the receptor Pdgfra, as well as the ligands Pdgfa and Pdgfb, were maintained in BM and spleen during ruxolitinib treatment. ${ }^{19}$ Additional experiments showed that PDGF signaling through MEK/ERK was not reduced upon ruxolitinib treatment. Combined treatment with JAK2 and MEK inhibitors was superior over inhibition with either compound alone in mouse models of Jak2 V617F and MPLW515-induced myelofibrosis, and reduced Pdgfra, Pdgfa, and Pdgfb transcript expression. These data suggest that combined MEK/JAK2 inhibition may be efficacious in treating MPN.

\section{Conclusions and future directions}

In conclusion, Kramer et al. have methodically and elegantly analyzed the sequential changes that occur in the BM during the initiation and progression of myelofibrosis in Gata1 ${ }^{\text {low }}$ mice and identified upregulation of the PDGF pathway as a hallmark of myelofibrosis. Their work suggests that increased PDGFR expression could be used as an early biomarker for myelofibrosis development. Given the paucity of reliable myelofibrosis biomarkers, this finding warrants further study in MPN patients. Additionally, now that next generation sequencing platforms are increasingly used to identify genetic predictors of progression to myelofibrosis in MPN, it would be interesting to study whether increased PDGF expression correlates with certain genetic subsets of MPN. Finally, given recent advancements enabling combined single-cell mutational and transcriptomic analyses, ${ }^{20}$ it will be possible to determine precisely which cellular sub-populations in the BM (both hematopoietic and stromal) are involved in PDGF signaling early in the course of myelofibrosis. Since a multitude of profibrotic factors are up-regulated in myelofibrosis, the therapeutic efficacy of inhibiting a single pathway, especially in advanced disease, may be limited. However, the identification and early targeting of pathways that are activated during the initial stages of myelofibrosis may prove more fruitful.

\section{Acknowledgments}

AM acknowledges support from the NIH (R01HL131835), the MPN Research Foundation and the Gabrielle's Angel Foundation for Cancer Research. Dr. Mullally is a Scholar of The Leukemia \& Lymphoma Society.

\section{References}

1. Kramer F, Dernedde J, Mezheyeuskiet A, et al. Platelet-derived growth factor receptor $\beta$ activation and regulation in murine myelofibrosis. Haematologica 2020;105(8):2083-2094

2. Shimizu R, Engel JD, Yamamoto M. GATA1-related leukaemias. Nat Rev Cancer. 2008; 8(4):279-287.

3. Vannucchi AM, Pancrazzi A, Guglielmelli P, et al. Abnormalities of GATA-1 in megakaryocytes from patients with idiopathic myelofibrosis. Am J Pathol. 2005;167(3):849-858.

4. Vannucchi AM, Bianchi L, Cellai C, et al. Development of myelofibrosis in mice genetically impaired for GATA-1 expression (GATA-1(low) mice). Blood. 2002;100(4):1123-1132.

5. Bock O, Loch G, Büsche G, von Wasielewski R, Schlué J, Kreipe H Aberrant expression of platelet-derived growth factor (PDGF) and PDGF receptor-alpha is associated with advanced bone marrow fibrosis in idiopathic myelofibrosis. Haematologica. 2005;90(1):133-134.

6. Bedekovics J, Kiss A, Beke L, Karolyi K, Méhes G. Platelet derived growth factor receptor-beta (PDGFRbeta) expression is limited to activated stromal cells in the bone marrow and shows a strong correlation with the grade of myelofibrosis. Virchows Arch. 2013;463(1):57-65.

7. Gersuk GM, Carmel R, Pattengale PK. Platelet-derived growth factor concentrations in platelet-poor plasma and urine from patients with myeloproliferative disorders. Blood. 1989;74(7):2330-2334.

8. Nurden AT, Nurden P. Inherited thrombocytopenias. Haematologica. 2007;92(9):1158-1164

9. Turro E, Greene D, Wijgaerts A, et al. A dominant gain-of-function mutation in universal tyrosine kinase SRC causes thrombocytopenia, myelofibrosis, bleeding, and bone pathologies. Sci Transl Med. 2016;8(328):328ra30.

10. Pardanani A, Tefferi A. Imatinib targets other than bcr/abl and their clinical relevance in myeloid disorders. Blood. 2004;104(7):1931-1939.

11. Cools J, DeAngelo DJ, Gotlib J, et al. A tyrosine kinase created by fusion of the PDGFRA and FIP1L1 genes as a therapeutic target of imatinib in idiopathic hypereosinophilic syndrome. $N$ Engl J Med. 2003;348(13):1201-1214.

12. Apperley JF, Gardembas M, Melo JV, et al. Response to imatinib mesylate in patients with chronic myeloproliferative diseases with rearrangements of the platelet-derived growth factor receptor beta. $\mathrm{N}$ Engl J Med. 2002;347(7):481-487.

13. Villeval JL, Cohen-Solal K, Tulliez M, et al. High thrombopoietin production by hematopoietic cells induces a fatal myeloproliferative syndrome in mice. Blood. 1997;90(11):4369-4383.

14. Decker M, Martinez-Morentin L, Wang G, et al. Leptin-receptorexpressing bone marrow stromal cells are myofibroblasts in primary myelofibrosis. Nat Cell Biol. 2017;19(6):677-688.

15. Papadopoulos N, Lennartsson J. The PDGF/PDGFR pathway as a drug target. Mol Aspects Med. 2018;62:75-88.

16. Marneth AE, Mullally A. The Molecular Genetics of Myeloproliferative Neoplasms. Cold Spring Harb Perspect Med. 2020;10(2):a034876.

17. Mesa RA. Imatinib and tyrosine kinase inhibition, in the management of BCR-ABL negative myeloproliferative disorders. Biologics. 2007;1(2):129-138.

18. Iurlo A, Gianelli U, Rapezzi D, et al. Imatinib and ruxolitinib association: first experience in two patients. Haematologica. 2014;99(6):e7677

19. Stivala $S$, Codilupi T, Brkic S, et al. Targeting compensatory MEK/ERK activation increases JAK inhibitor efficacy in myeloproliferative neoplasms. J Clin Invest. 2019;129(4):1596-1611.

20. Psaila B, Wang G, Rodriguez-Meira A, et al. Single-Cell Analyses Reveal Megakaryocyte-Biased Hematopoiesis in Myelofibrosis and Identify Mutant Clone-Specific Targets. Mol Cell. 2020;78(3):477492.e8. 